\title{
EMBEDDING PUNCTURED MANIFOLDS
}

D. B. A. EPSTEIN

Let $M$ be a compact differential manifold without boundary of dimension $m$.

Theorem. Let $M-x$ be embedded differentially in a differential manifold $N$ of dimension $n$, in such a way that the normal bundle of the embedding is fibre homotopically trivial. Then there is a map of degree one onto the smash product $N \rightarrow S^{n-m} \wedge M$. (The degree should be taken mod 2 if $M$ or $N$ is not orientable, and with respect to the compact cohomology of $N-\mathrm{Bd} N$ if $N$ is not compact or has boundary.)

Proof. Let $D^{m}$ be a closed disk in $M$ such that $x \in D^{m} \subset M$. The normal $(n-m)$-dimensional disk bundle of $M-\operatorname{Int} D^{m}$ in $M$ has as its total space a compact $n$-dimensional manifold $L$ with boundary. Let $K=L / \operatorname{Bd} L . L$ can be regarded as a submanifold of $N$ by the tubular neighbourhood theorem. The map $N \rightarrow K$, which sends $N-L$ to a point and which sends $L \rightarrow K$ via the identification map, has degree one. We shall show that $K$ is homotopy equivalent to $S^{n-m} \wedge M$.

Let $L_{1}$ be the total space of the trivial $(n-m)$-dimensional disk bundle over $M-\operatorname{Int} D^{m}$. Let $K_{1}=L_{1} / \mathrm{Bd} L_{1}$. The fibre homotopy equivalence, referred to in the hypotheses, gives rise to a homotopy equivalence between $K$ and $K_{1}$. Now

$$
\begin{aligned}
K_{1} & =M \times D^{n-m} / D^{m} \times D^{n-m} \cup M \times S^{n-m-1} \\
& =M \times S^{n-m} / D^{m} \times S^{n-m} \cup M \times * \\
& =M \times S^{n-m} / * \times S^{n-m} \cup M \times * \\
& =M \wedge S^{n-m} .
\end{aligned}
$$

This proves the theorem.

Corollary 1. If $M=L(2 n, q)$, the three-dimensional lens space, then $M-x$ cannot be differentially embedded in $S^{4}$.

Proof. If $M-x$ is embedded in $S^{4}$, then, by the theorem, there is a map of degree one

$$
S^{4} \rightarrow S^{1} \wedge M
$$

But Puppe $[1$, p. 416], shows that this is not true. (Puppe shows that

Received by the editors October 18, 1963. 
a certain cohomology operation from dimension two to dimension four in $S^{1} \# M$ is nonzero.) This completes the proof.

Zeeman [2] has shown that if $M=L(2 n+1, q)$, then $M-x$ can be differentially embedded in $S^{4}$. It is well known that no lens space can be embedded in $S^{4}$ and any orientable three-dimensional manifold can be embedded in $S^{5}$ [4]. The situation with regard to embeddings of lens spaces and punctured lens spaces is therefore completely solved.

CoROllary 2. Let $M$ be a simply connected, stably parallelizable manifold. Let $M-x$ be differentially embedded in $S^{n}$, where $2 n \geqq 3(m+1)$ and let the normal bundle of the embedding be fibre homotopically trivial. Then $M$ can be differentially embedded in $S^{n}$ (and we can assume that the normal bundle of the embedding is fibre homotopically trivial).

Proof. By the theorem, we have a map of degree one

$$
S^{n} \rightarrow S^{n-m} \wedge M
$$

Now $S^{n-m-1} * M$ is homotopy equivalent to $S^{n-m} \wedge M$ and there is a map of degree one of $S^{n-m-1} * M$ onto the Thom space of the trivial $(n-m)$-dimensional vector bundle over $M$. We therefore have a map of degree one of $S^{n}$ onto the Thom space of the trivial bundle over $M$.

We have therefore verified all the hypotheses of a theorem proved by Levine [3, Theorem 1], the conclusion of which is the same as the conclusion of our Corollary 2.

The author would like to thank C. T. C. Wall and D. Puppe for helpful conversations.

\section{BIBLIOGRAPHY}

1. D. Puppe, Homotopiemengen und ihre induzierten Abbildungen. II, Math. Z. 69 (1958), 395-417.

2. E. C. Zeeman, On twisting spun knots, Trans. Amer. Math. Soc. (1965), (to appear).

3. J. Levine, On differentiable imbeddings of simply-connected manifolds, Bull. Amer. Math. Soc. 69 (1963), 806-809.

4. M. W. Hirsch, The imbedding of bounding manifolds in Euclidean space, Ann. of Math. 74 (1961), 494-497.

Churchill College, Cambridge 